\title{
Desorption Kinetics and Binding Energies of Small Hydrocarbons
}

\author{
Aida Behmard ${ }^{1}(1)$, Edith C. Fayolle ${ }^{2,3}$ (), Dawn M. Graninger ${ }^{4}$ (1), Jennifer B. Bergner ${ }^{2}$ (1), Rafael Martín-Doménech ${ }^{2}$, \\ Pavlo Maksyutenko ${ }^{2}$, Mahesh Rajappan ${ }^{2}$, and Karin I. Öberg ${ }^{2}$ (D) \\ ${ }^{1}$ Division of Geological and Planetary Sciences, California Institute of Technology, Pasadena, CA 91125, USA \\ ${ }^{2}$ Harvard-Smithsonian Center for Astrophysics, 60 Garden Street, Cambridge, MA 02138, USA \\ ${ }^{3}$ Jet Propulsion Laboratory, California Institute of Technology, 4800 Oak Grove Drive, Pasadena, CA 91109-8099, USA \\ ${ }^{4}$ Lawrence Livermore National Laboratory, 7000 East Avenue, Livermore, CA 94550, USA \\ Received 2018 August 29; revised 2019 March 6; accepted 2019 March 8; published 2019 April 17
}

\begin{abstract}
Small hydrocarbons are an important organic reservoir in protostellar and protoplanetary environments. Constraints on desorption temperatures and binding energies of such hydrocarbons are needed for accurate predictions of where these molecules exist in the ice versus gas phase during the different stages of star and planet formation. Through a series of temperature programmed desorption experiments, we constrain the binding energies of 2- and 3-carbon hydrocarbons $\left(\mathrm{C}_{2} \mathrm{H}_{2}\right.$ - acetylene, $\mathrm{C}_{2} \mathrm{H}_{4}$ - ethylene, $\mathrm{C}_{2} \mathrm{H}_{6}$-ethane, $\mathrm{C}_{3} \mathrm{H}_{4}$ - propyne, $\mathrm{C}_{3} \mathrm{H}_{6}$ - propene, and $\mathrm{C}_{3} \mathrm{H}_{8}$ - propane) to $2200-4200 \mathrm{~K}$ in the case of pure amorphous ices, to $2400-4400 \mathrm{~K}$ on compact amorphous $\mathrm{H}_{2} \mathrm{O}$, and to $2800-4700 \mathrm{~K}$ on porous amorphous $\mathrm{H}_{2} \mathrm{O}$. The 3-carbon hydrocarbon binding energies are always larger than the 2-carbon hydrocarbon binding energies. Within the 2- and 3-carbon hydrocarbon families, the alkynes (i.e., least-saturated) hydrocarbons exhibit the largest binding energies, while the alkane and alkene binding energies are comparable. Binding energies are $~ 5 \%-20 \%$ higher on water ice substrates compared to pure ices, which is a small increase compared to what has been measured for other volatile molecules such as $\mathrm{CO}$ and $\mathrm{N}_{2}$. Thus in the case of hydrocarbons, $\mathrm{H}_{2} \mathrm{O}$ has a less pronounced effect on sublimation front locations (i.e., snowlines) in protoplanetary disks.
\end{abstract}

Key words: astrochemistry - methods: laboratory: molecular - protoplanetary disks

\section{Introduction}

Simple hydrocarbons are common in protostellar and circumstellar environments (Tucker et al. 1974; Betz 1981; Öberg et al. 2008; Pontoppidan et al. 2014; Guzmán et al. 2015), and may constitute an important reservoir of volatile carbon during planet formation. In solar system comets, which are thought to preserve the volatile composition of the outer solar nebula, hydrocarbon detections include $\mathrm{CH}_{4}, \mathrm{C}_{2} \mathrm{H}_{2}$, and $\mathrm{C}_{2} \mathrm{H}_{6}$ (Brooke et al. 1996; Mumma et al. 1996; Hudson \& Moore 1997; Kawakita et al. 2014). Hydrocarbons have also been detected in solar nebula analogs: $\mathrm{C}_{2} \mathrm{H}$ and $c-\mathrm{C}_{3} \mathrm{H}_{2}$ at millimeter/submillimeter wavelengths, $\mathrm{CH}_{4}$ in the near-IR, $\mathrm{C}_{2} \mathrm{H}_{2}$ in the mid- and near-IR, and the hydrocarbon radical $\mathrm{CH}^{+}$in the far-IR (Lahuis et al. 2006; Gibb et al. 2007; Thi et al. 2011; Gibb \& Horne 2013; Qi et al. 2013; Pontoppidan et al. 2014; Kastner et al. 2015). Larger hydrocarbons, such as $\mathrm{C}_{3} \mathrm{H}_{4}$ and $\mathrm{C}_{3} \mathrm{H}_{6}$, are expected to be present in disks and comets because they are frequently observed during early stages of star formation (Markwick et al. 2002), but have yet to be detected (Snyder \& Buhl 1973).

In interstellar and circumstellar environments, hydrocarbons can form through several pathways. During the early stages of cloud formation, when the majority of carbon in the gas phase is in the form of atomic carbon, unsaturated hydrocarbons form efficiently through ion-molecule gas-phase chemistry (e.g., Agúndez \& Wakelam 2013). These carbon atoms can also adsorb onto grain surfaces where hydrogen addition to adsorbed atomic carbon leads to the formation of $\mathrm{CH}_{4}$ (e.g., Tielens \& Hagen 1982). $\mathrm{CH}_{4}$ and other small hydrocarbons can then serve as starting points for larger hydrocarbon formation, both through grain surface reactions (Moore \& Hudson 1998; Öberg et al. 2010), and through gas-phase reactions following desorption (Charnley 2004; Sakai \& Yamamoto 2013). Warm carbon chain chemistry is initiated by $\mathrm{CH}_{4}$ sublimation from icy grain mantles and leads to the formation of long, unsaturated carbon chains (Sakai \& Yamamoto 2013; Graninger et al. 2016). In the solid state, hydrocarbon-rich ices are proposed to be the starting point of a rich prebiotic chemistry (Kaiser \& Roessler 1998; Bernstein et al. 2005; Hardegree-Ullman et al. 2014). Gas-phase reactions are proposed as a major source of hydrocarbons in the envelopes of protostars (Sakai \& Yamamoto 2013; Graninger et al. 2016).

Hydrocarbons formed during the pre- and protostellar stages of star formation are likely inherited by the protoplanetary disk. In the disk, hydrocarbon chemistry may proceed to produce a new, distinct set of products, though the relative importance of inheritance and in situ chemistry for organic molecules is still debated (e.g., Cleeves et al. 2016). In either case, predicting how hydrocarbons are incorporated into plantestimals and planetary atmospheres requires understanding their division between gas and ice phases throughout protoplanetary disks. This division is set by the locations of hydrocarbon sublimation fronts, and by the ease with which hydrocarbons are entrapped in less volatile ices. Both are important to quantify. This study addresses the former process.

The sublimation front locations of molecular species are dictated by adsorption and desorption kinetics, which are in turn set by their binding energies (e.g., Viti et al. 2004; Hollenbach et al. 2009; He et al. 2016, 2017). Some constraints on binding energies exist for $\mathrm{CH}_{4}, \mathrm{C}_{2} \mathrm{H}_{2}, \mathrm{C}_{2} \mathrm{H}_{6}$, and $\mathrm{C}_{3} \mathrm{H}_{8}$ from different experimental studies (Collings et al. 2004; He et al. 2016; Smith et al. 2016). We expand on these through a survey of desorption behavior for all linear 2-3 carbon hydrocarbons. Both pure ice desorption and desorption off $\mathrm{H}_{2} \mathrm{O}$ substrates are investigated. Among these experiments, desorption off compact $\mathrm{H}_{2} \mathrm{O}$ ice is the most relevant for the majority of 
astrophysical environments (Boogert et al. 2015). In Section 2 we present the experimental setup and methods used to characterize hydrocarbon desorption. The experimental results and the binding energies are presented in Section 3 and discussed in Section 4, and some astrophysical implications are presented in Section 5.

\section{Methods}

\subsection{Experimental Details}

TPD experiments were conducted in an ultra-high vacuum (UHV) chamber described in detail by Lauck et al. (2015). The UHV chamber has a base pressure of $<5 \times 10^{-10}$ Torr at room temperature, dominated by $\mathrm{H}_{2}$. Amorphous ices were grown on a 0.75 inch diameter and $2 \mathrm{~mm}$ thick Cs I window at the center of the chamber that can be cooled to $11 \mathrm{~K}$ by a closed-cycle $\mathrm{He}$ cryostat. Unless otherwise noted, all ices used in TPD experiments throughout this study are amorphous in structure, which is most relevant for interstellar environments (Hagen et al. 1981; Oba et al. 2009). Gaseous $\mathrm{C}_{2} \mathrm{H}_{2}$-acetylene (99.6\% Matheson Trigas) ${ }^{5}, \mathrm{C}_{2} \mathrm{H}_{4}$ - ethylene (99.99\% Sigma-Aldrich), $\mathrm{C}_{2} \mathrm{H}_{6}$ - ethane (99.99\% Sigma-Aldrich), $\mathrm{C}_{3} \mathrm{H}_{4}$-propyne ( $\geqslant 99 \%$ Sigma-Aldrich), $\mathrm{C}_{3} \mathrm{H}_{6}$-propene ( $\geqslant 99 \%$ Sigma-Aldrich), and $\mathrm{C}_{3} \mathrm{H}_{8}$-propane (99.97\% Sigma-Aldrich) were deposited through a $4.8 \mathrm{~mm}$ diameter pipe with the outlet located 0.8 inches from the Cs I window onto the bare $11 \mathrm{~K} \mathrm{Cs} \mathrm{I} \mathrm{window} \mathrm{or} \mathrm{onto} \mathrm{thick}$ amorphous compact/porous $\mathrm{H}_{2} \mathrm{O}$ ice substrates. The $\mathrm{H}_{2} \mathrm{O}$ was purified beforehand through at least three freeze-pump-thaw cycles using liquid nitrogen. $\mathrm{H}_{2} \mathrm{O}$ substrates were grown by depositing deionized $\mathrm{H}_{2} \mathrm{O}$ at $100 \mathrm{~K}$ for compact substrates, followed by cool-down to $11 \mathrm{~K}$, and at $11 \mathrm{~K}$ for porous substrates. Deposition temperature influences ice structure (compact versus porous) due to molecular rearrangement resulting from thermal diffusion (e.g., Bossa et al. 2012; Clements et al. 2018). The degree of porosity can be determined from the intensity of the dangling $\mathrm{O}-\mathrm{H}$ bond spectral feature (Devlin \& Buch 1995). However, we were unable to investigate the porosity of our water films via the dangling $\mathrm{O}-\mathrm{H}$ bond as it is not well detected in our ices (see Figure 9, left panel, in the Appendix; the dangling $\mathrm{O}-\mathrm{H}$ bond would be visible at $\sim 3600-3700 \mathrm{~cm}^{-1}$ ). This indicates that the porosity of our porous water films is low, but does not indicate a total lack of porosity (Raut et al. 2007; Isokoski et al. 2014). Laboratory studies also show that the dangling $\mathrm{O}-\mathrm{H}$ bond cannot be used to investigate porosity quantitatively (e.g., Bossa et al. 2014). To investigate this further, we conducted additional TPD experiments of $\mathrm{CO}$ on compact and porous $\mathrm{H}_{2} \mathrm{O}$ substrates, and found that the TPD curve of the porous experiment exhibits a $\mathrm{CO}$ desorption peak around the $\mathrm{H}_{2} \mathrm{O}$ desorption temperature, while that of the compact experiment does not (see Figure 9, right panel, in the Appendix). This provides additional evidence that our porous substrates do indeed contain pores. We confirmed that the $\mathrm{H}_{2} \mathrm{O}$ ices were amorphous rather than crystalline in structure from the shapes of the $\mathrm{H}_{2} \mathrm{O}$ IR spectral bands (e.g., Mastrapa et al. 2009).

Following deposition, infrared spectra of ice films were taken using a Fourier transform infrared spectrometer (FTIR, Bruker

\footnotetext{
The $\mathrm{C}_{2} \mathrm{H}_{2}$ was dissolved in acetone, accounting for its relatively low purity. One should note that we used $\mathrm{C}_{2} \mathrm{H}_{2}$ straight from the bottle without any purification steps because no acetone was detected by quadrupole mass spectrometer analysis during deposition, and no acetone IR features were observed in the FTIR spectrum of deposited $\mathrm{C}_{2} \mathrm{H}_{2}$.
}

Vertex 70v) in transmission mode. To produce a single spectrum, 128 scans were averaged over the $4000-600 \mathrm{~cm}^{-1}$ spectrometer range at a resolution of $1 \mathrm{~cm}^{-1}$. TPD measurements were performed by linearly heating the prepared ices at $2 \mathrm{~K}$ minutes $^{-1}$ and monitoring the desorbing molecules using a quadrupole mass spectrometer (QMS, Pfeiffer QMG 220M1) until complete hydrocarbon desorption. The Cs I window temperature was monitored and increased using a temperature controller (LakeShore $355)$ that operates a heating element situated above the window holder and silicon diode sensors attached onto the window holder. The measured temperature has an estimated accuracy of $2 \mathrm{~K}$ and a relative uncertainty of $0.1 \mathrm{~K}$. We obtained TPD plots in desorbing molecules $\mathrm{K}^{-1}$ by scaling the main hydrocarbon fragment ion current from the QMS using factors derived from methods explained in Section 2.2.

\subsection{Ice Thicknesses}

This study presents data on pure hydrocarbon ice desorption, and hydrocarbon desorption off compact and porous $\mathrm{H}_{2} \mathrm{O}$ ice. Pure hydrocarbon desorption requires ice thicknesses greater than a few monolayers to ensure that the initial desorption curve is dominated by hydrocarbonhydrocarbon interactions. In the cases of desorption off $\mathrm{H}_{2} \mathrm{O}$ substrates, ice thicknesses should be in the mono- or submonolayer regime where the kinetics are dominated by hydrocarbon- $\mathrm{H}_{2} \mathrm{O}$ interactions. Ice thickness measurements are required to first ensure that the experiments are carried out in the correct desorption regime, and later to extract binding energies from TPD curves. We also use the estimated ice thicknesses for each experiment to convert the QMS ion current to a desorption rate in units of molecules $\mathrm{K}^{-1}$.

We used three different methods to determine hydrocarbon ice thicknesses: IR spectroscopy (for the pure $\mathrm{C}_{2} \mathrm{H}_{x}$ ices), integrated ion currents from the TPD experiments (for the pure $\mathrm{C}_{3} \mathrm{H}_{x}$ ices and all hydrocarbons desorbing off compact water), and TPD shape characteristics (for all hydrocarbons desorbing off porous water). Errors on ice thicknesses determined from any method other than IR band strengths are taken as $50 \%$. The ice thicknesses are given in monolayer units with the typical approximation of $1 \mathrm{ML}=10^{15}$ molecules $\mathrm{cm}^{-2}$. However, $1 \mathrm{ML}$ does not always correspond to one molecular layer; porous surfaces are rougher than compact surfaces, allowing them to accommodate more molecules upon a surface area unit. At the same time, porous surfaces contain pores that can trap molecules and inhibit their release via desorption. By comparing thicknesses calculated from IR band strengths versus using porous experiments as thickness calibrations, we found that these effects roughly cancel each other out, and that the porous $\mathrm{H}_{2} \mathrm{O}$ TPDs provide a reasonable measure of the ion current corresponding to 1 ML.

To determine pure $\mathrm{C}_{2} \mathrm{H}_{x}$ ice thicknesses with IR spectroscopy, we used post-deposition IR spectra and hydrocarbon band strengths. Band strengths relevant for the $\mathrm{C}_{2} \mathrm{H}_{x}$ hydrocarbons in our set are reported in Hudson et al. (2014a, 2014b), and Gerakines et al. (1995; Figure 1, left panel). All IR modes and associated band strengths used in this work are reported in Table 1. Thicknesses were calculated from the formula:

$$
N_{i}=\frac{\ln (10) \int I(\nu) d \nu}{A_{i}},
$$



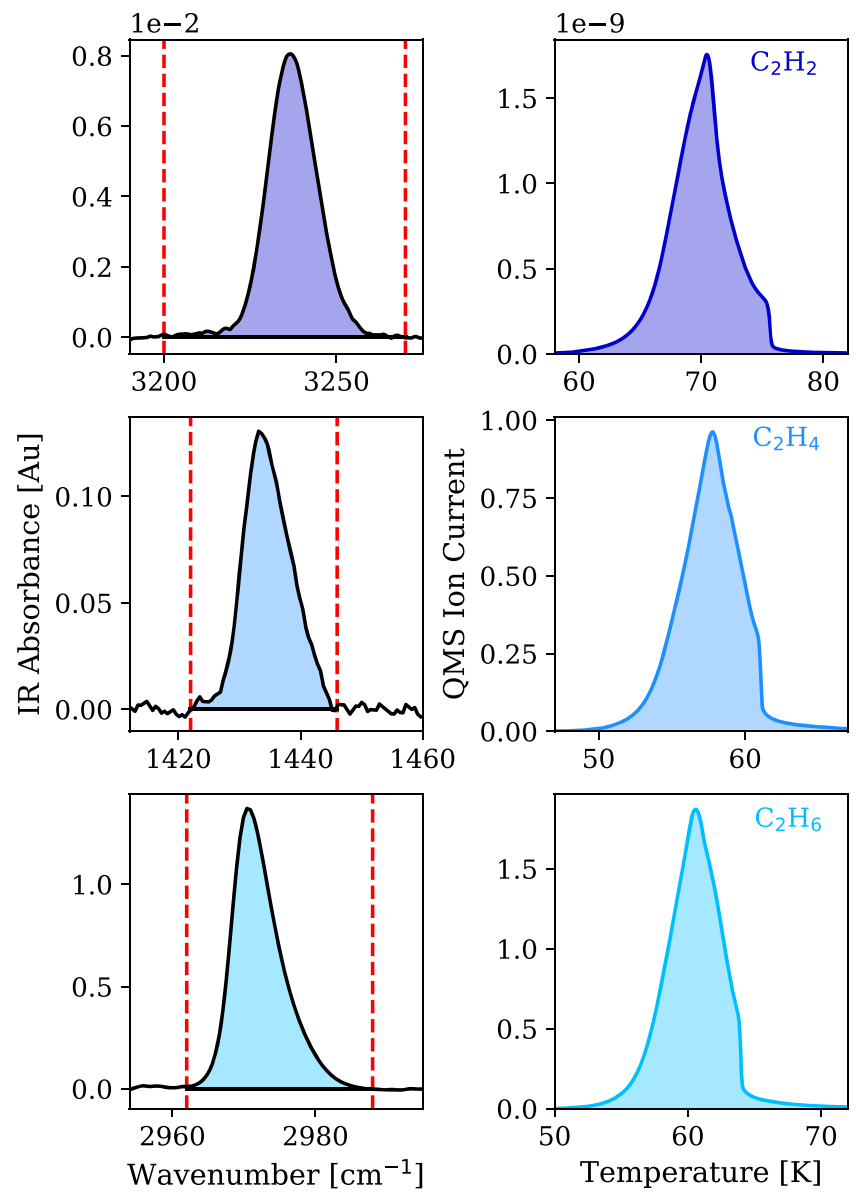

Figure 1. Left column displays the strongest IR features that were used to estimate the ice thicknesses for each pure $\mathrm{C}_{2} \mathrm{H}_{x}$ TPD, with the shaded regions identifying the integrated regions. The right column displays the pure 2-carbon TPDs that were used as references to estimate the thicknesses of $\mathrm{C}_{2} \mathrm{H}_{x}$ TPDs on compact water substrates.

Table 1

IR Band Positions and Strengths

\begin{tabular}{lccc}
\hline \hline Molecule & IR Mode & $\begin{array}{c}\text { Position } \\
\left(\mathrm{cm}^{-1}\right)\end{array}$ & $\begin{array}{c}\text { Band Strength } A_{i} \\
(\mathrm{~cm} \mathrm{molecule})\end{array}$ \\
\hline $\mathrm{H}_{2} \mathrm{O}$ & $\nu_{1}$ & 3280 & $2.0 \times 10^{-16}$ \\
$\mathrm{C}_{2} \mathrm{H}_{2}$ & $\nu_{5}$ & 3240 & $2.39 \times 10^{-17}$ \\
$\mathrm{C}_{2} \mathrm{H}_{4}$ & $\nu_{7}$ & 1434.3 & $2.24 \times 10^{-18}$ \\
$\mathrm{C}_{2} \mathrm{H}_{6}$ & $\nu_{5}$ & 2972.3 & $2.20 \times 10^{-17}$ \\
\hline
\end{tabular}

Note. Errors on all band strengths are uniformly set at $20 \%$. For justification, see Section 2.2.

where $N_{i}$ is the column density (molecule $\mathrm{cm}^{-2}$ ), $\int I(\nu) d \nu$ is the integrated area of the IR band (absorbance units), and $A_{i}$ is the band strength in optical depth units as reported in the literature. Though reported band strength errors are between $0.5 \%$ and $6 \%$, we adopted an error of $20 \%$ on all band strengths to account for possible differences in temperature and ice structure between our study and those from which the band strengths were extracted. A $20 \%$ error is also consistent with the variance in ice thickness measurements we obtain when different IR bands are chosen for the calculation.

Pure $\mathrm{C}_{3} \mathrm{H}_{x}$ ice thicknesses could not be measured with IR spectroscopy due to the lack of $\mathrm{C}_{3} \mathrm{H}_{x}$ band strengths reported in
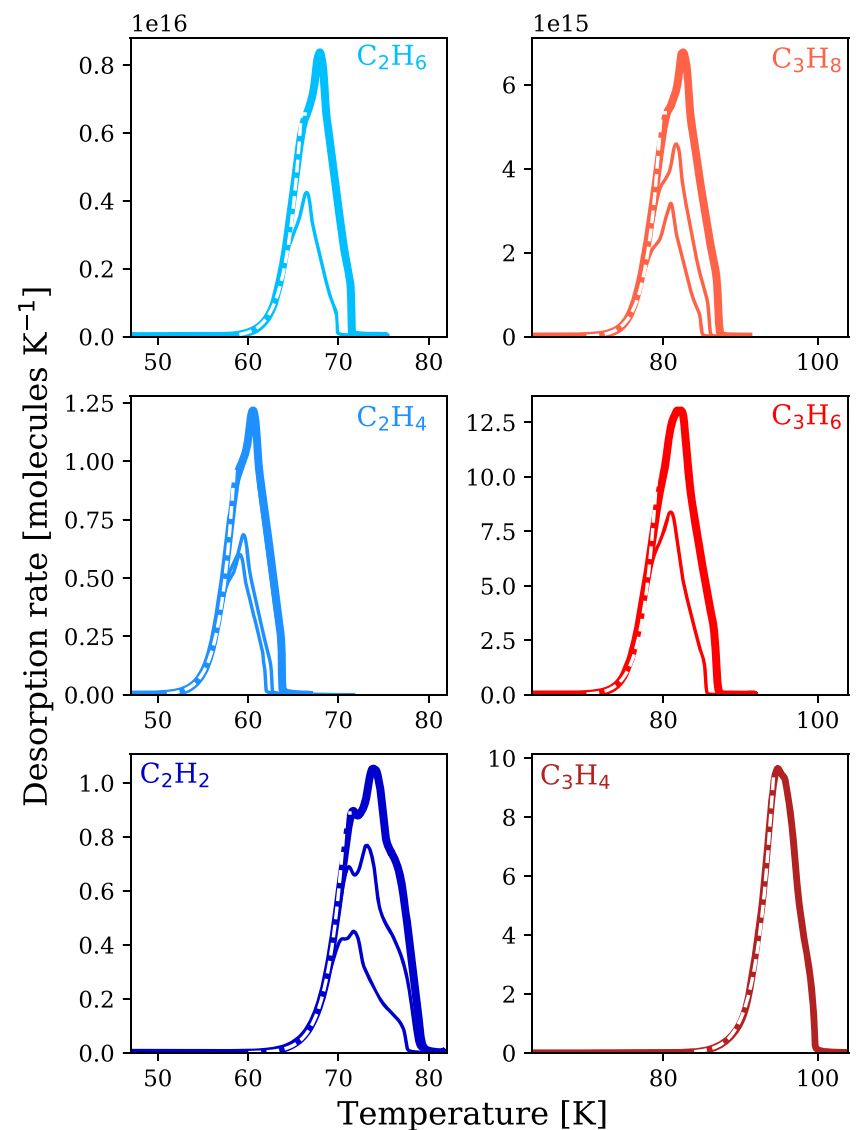

Figure 2. Pure 2-carbon (left) and 3-carbon (right) hydrocarbon TPD curves displayed in solid colored lines, with overlaid white dashed lines representing the fit to obtain the binding energies. When available, TPD runs of thinner ices are overlaid in thinner solid colored lines to demonstrate that the zeroth-order regime was achieved. In the case of $\mathrm{C}_{3} \mathrm{H}_{4}$, the first TPD curve we acquired clearly showed zeroth-order kinetics and we therefore did not gather additional, supporting data.

the literature. Instead, they are estimated from their integrated TPD curves using the $\mathrm{C}_{3} \mathrm{H}_{x}$ porous experiments as references, which are assumed to have thicknesses of $\sim 1$ ML (justified below). In any case, to ensure that the pure ice experiments (both 2- and 3-carbon) were in the multilayer regime where energies are independent of thickness, we ran a series of TPD experiments of increasing thickness and checked for overlapping leading edges (Figure 2).

We estimated the thicknesses of ices (both 2- and 3-carbon hydrocarbons) on porous $\mathrm{H}_{2} \mathrm{O}$ by noting that the TPD curves of all experiments involving porous substrates deviate from the profile expected for pure submonolayer coverages by exhibiting a small multilayer peak, indicating that they are on the verge of reaching the multilayer desorption regime and thus correspond to $\sim 1 \mathrm{ML}$ coverage (Figure 3).

For experiments of hydrocarbon desorption off compact $\mathrm{H}_{2} \mathrm{O}$, we aimed to deposit submonolayer coverages of hydrocarbon ice onto a $\sim 50 \mathrm{ML} \mathrm{H}_{2} \mathrm{O}$ ice substrate. The hydrocarbon ice thicknesses could not be verified with IR spectroscopy because IR bands of thin ices are weak and broad in the presence of $\mathrm{H}_{2} \mathrm{O}$. To obtain ice thicknesses for $\mathrm{C}_{2} \mathrm{H}_{x}$ ices on compact substrates, we compared the integrated areas of their TPD curves (ion current in $A \cdot s$ ) to those of the pure ice experiments, and multiplied the integrated TPD ratios with the known ice thicknesses of the pure experiments. (Figure 1, right panel; e.g., Bertin et al. 2011; Doronin et al. 2015). This 
method assumes that the QMS signal is proportional to the number of desorbing molecules and that the chamber vacuum pump is evacuating gas at a high speed, both of which have been experimentally verified. Because the $\mathrm{C}_{3} \mathrm{H}_{x}$ band strengths are unknown, we could not use this procedure to estimate the thicknesses of 3-carbon ices on compact substrates. Instead, we used the integrated ion current ratios with the 3-carbon ices on porous substrates as references, as in the case of determining the pure 3-carbon ice thicknesses.

We chose compact ice experiments with coverages of $\sim 0.2$ ML to ensure that we were in the regime where hydrocarbon- $\mathrm{H}_{2} \mathrm{O}$ interactions dominate. Ideally, we wanted the thinnest coverages possible to isolate these interactions, but found that desorption of films thinner than $\sim 0.2 \mathrm{ML}$ did not produce detectable signals in the TPD data. Our choice of $\sim 0.2$ ML coverages on compact substrates is further discussed in Section 3.2.

\subsection{Modeling}

To obtain binding energies, we fit the TPD curves with the Polanyi-Wigner equation:

$$
-\frac{d \theta}{d T}=\frac{\nu}{\beta} \theta^{n} e^{-E_{b} / T},
$$

where $n$ is the desorption order, $\theta$ is the ice coverage, $T$ is the temperature in $\mathrm{K}, \nu$ is a pre-exponential factor in $\mathrm{ML}^{(1-n)} \mathrm{s}^{-1}$, $\beta$ is the heating rate in $\mathrm{K} \mathrm{s}^{-1}$, and $E_{b}$ is the binding energy in $\mathrm{K}$.

For pure ices, we determined the hydrocarbon binding energies by fitting the TPD curves using zeroth-order kinetics $\left(n=0\right.$ in Equation (1)). We calculated $E_{b}$ and the preexponential factor $\nu$ simultaneously by fitting the logarithm of the desorption rate versus the inverse of the temperature with a straight line. The process is illustrated in Figure 10 in the Appendix.

The hydrocarbon- $\mathrm{H}_{2} \mathrm{O}$ TPDs are fitted with the first-order ( $n=1$ in Equation (1)) form of the Polanyi-Wigner equation as is appropriate for submonolayer desorption where the ice system is characterized by a single binding energy. The nonhomogeneous nature of amorphous water ice results in surfaces with a range of binding sites. We therefore fit the submonolayer interaction of the curve with a distribution of binding energies described by a linear combination of firstorder desorption kinetics (Noble et al. 2012; Collings et al. 2015; Doronin et al. 2015; Fayolle et al. 2016). This is accomplished by sampling a range of binding energies in steps of $60-100 \mathrm{~K}$. We used a range of $1800-3700 \mathrm{~K}$ for 2-carbon hydrocarbons and $3900-5500 \mathrm{~K}$ for 3-carbon hydrocarbons on compact substrates, and a range of $2700-5000 \mathrm{~K}$ for 2-carbon hydrocarbons and $3000-5500 \mathrm{~K}$ for 3-carbon hydrocarbons on porous substrates. An alternative method for modeling submonolayer desorption is presented in Smith et al. (2016), which differs from our approach by modeling the binding energy as a continuous function of coverage. However, we note that our chosen step sizes are well within the binding energy errors and should thus not be a major contributor to the binding energy distribution uncertainties.

We obtain a binding energy distribution from which a mean $E_{b}$ and associated FWHM can be extracted assuming a Gaussian distribution. Pre-exponential factors $\nu$ derived from the multilayer regime calculations are used in the monolayer and submonolayer regime calculations. For more details on the binding energy calculation procedures, see
Fayolle et al. (2016). Errors on calculations take into account the estimated accuracy of $2 \mathrm{~K}$ and relative uncertainty of $0.1 \mathrm{~K}$ on the temperature instrument as well as errors on ice thickness (see Section 3.2).

\section{Results}

\subsection{Temperature Programmed Desorption Curves}

A summary of all experiments is provided in Table 2. TPD curves on $\mathrm{Cs} \mathrm{I}$, compact $\mathrm{H}_{2} \mathrm{O}$, and porous $\mathrm{H}_{2} \mathrm{O}$ substrates are displayed in Figure 4.

Pure Hydrocarbon Ices. On Cs I substrates, 2-carbon hydrocarbons all exhibit lower desorption temperatures than 3-carbon hydrocarbons. Within the 2-carbon hydrocarbon set, $\mathrm{C}_{2} \mathrm{H}_{4}$ exhibits the lowest desorption temperature, followed by $\mathrm{C}_{2} \mathrm{H}_{6}$ and lastly $\mathrm{C}_{2} \mathrm{H}_{2}$. The same desorption temperature trend of alkene, alkane, alkyne is exhibited within the 3-carbon hydrocarbon set, with $\mathrm{C}_{3} \mathrm{H}_{6}$ exhibiting the lowest desorption temperature, followed by $\mathrm{C}_{3} \mathrm{H}_{8}$ and $\mathrm{C}_{3} \mathrm{H}_{4}$ (Figure 2).

The TPD curves of 2-carbon hydrocarbons and $\mathrm{C}_{3} \mathrm{H}_{8}$ exhibit fall off from the initial leading edge, resulting in "bump"-like features (Figure 4). These "bumps" may be due to an amorphous-to-crystalline transition. There are studies that have constrained the temperatures at which amorphous-to-crystalline transitions are expected to occur for 2-carbon hydrocarbons (e.g., Anderson et al. 1985; Khanna et al. 1988; Zhao et al. 1988; Hudson et al. 2014a, 2014b), and these are generally consistent with the temperature points of the "bumps" in our pure 2-carbon TPD curves (Figure 2). Whether the bumps observed in the 3-carbon TPDs also coincide with phase changes is unclear due to a lack of experimental data. We did not monitor the ices with the FTIR during warm-up and cannot confirm that there is indeed a crystalline phase transition for any of the experiments.

Thin Ices on Porous $\mathrm{H}_{2} \mathrm{O}$ Substrates. All of the hydrocarbons on porous $\mathrm{H}_{2} \mathrm{O}$ present both a multilayer and a submonolayer peak, indicating that the targeted $\sim 1 \mathrm{ML}$ coverage is achieved. As in the pure ice experiments, 2-carbon hydrocarbons have lower desorption temperatures than 3-carbon hydrocarbons. However, the desorption temperature trend changes from that of the pure ice set to alkane, alkene, alkyne for both the 2- and 3-carbon hydrocarbon compact sets. Compared to the compact ice experiments, the porous ice TPD curves appear broader, but as the ice coverages are different no direct comparison is possible. All TPD curves display another large peak near the $\mathrm{H}_{2} \mathrm{O}$ desorption temperature ( 140-160 K) due to release of entrapped molecules (not shown here).

Thin Ices on Compact $\mathrm{H}_{2} \mathrm{O}$ Substrates. Multiple TPD runs of each hydrocarbon on compact $\mathrm{H}_{2} \mathrm{O}$ substrates were taken to explore the effects of ice coverage around the target coverage of $\sim 0.2 \mathrm{ML}$.

For the alkanes and alkynes, increasing coverage does not produce the double-peaked desorption curve characteristic of a submonolayer that is more strongly bound than subsequent layers. This is in contrast to the alkenes where a lower temperature desorption peak does appear for the thickest coverages, which can be associated with multilayer desorption (Figure 4). The lack of such a peak for the alkanes and alkynes indicates that the water-hydrocarbon and hydrocarbonhydrocarbon interactions are of comparable strengths. This is confirmed by the differences in binding energies for pure alkanes and alkynes, which differ from the binding energies of 
Table 2

Experimental Summary: Binding Energies for Pure Ice Multilayer Regime TPDs and Mean Binding Energies with FWHMs for Monolayer and Submonolayer Regime TPDs on $\mathrm{H}_{2} \mathrm{O}$ Substrates (FWHMs Indicated in Brackets)

\begin{tabular}{|c|c|c|c|c|c|}
\hline Species & Substrate & $\begin{array}{l}\text { Ice Thickness } \\
\text { (ML) }\end{array}$ & $\begin{array}{l}T_{\text {des }} \\
(\mathrm{K})\end{array}$ & $\begin{array}{c}\nu \\
\left(\mathrm{s}^{-1}\right)\end{array}$ & $\begin{array}{c}E_{b} \\
(\mathrm{~K})\end{array}$ \\
\hline $\mathrm{C}_{2} \mathrm{H}_{2}$ & Cs I Window & $77 \pm 15^{\mathrm{a}}$ & 74 & $3_{-2.5}^{+17} \times 10^{16}$ & $2800_{-300}^{+200}$ \\
\hline $\mathrm{C}_{2} \mathrm{H}_{4}$ & Cs I Window & $59 \pm 12^{\mathrm{a}}$ & 61 & $4_{-3.5}^{+36} \times 10^{15}$ & $2200_{-100}^{+200}$ \\
\hline $\mathrm{C}_{3} \mathrm{H}_{4}$ & Cs I Window & $\sim 50^{\mathrm{b}}$ & 95 & $1_{-0.8}^{+4} \times 10^{19}$ & $4200_{-200}^{+300}$ \\
\hline $\mathrm{C}_{3} \mathrm{H}_{6}$ & Cs I Window & $\sim 90^{\mathrm{b}}$ & 82 & $6_{-5.1}^{+34} \times 10^{18}$ & $3500_{-300}^{+300}$ \\
\hline $\mathrm{C}_{2} \mathrm{H}_{4}$ & Compact $\mathrm{H}_{2} \mathrm{O}$ & $\sim 0.2(0.1,0.4)^{\mathrm{b}}$ & 60 & $4_{-3.5}^{+36} \times 10^{15 c}$ & $2400_{-5}^{+5}[160](2460,2360)$ \\
\hline $\mathrm{C}_{2} \mathrm{H}_{6}$ & Compact $\mathrm{H}_{2} \mathrm{O}$ & $\sim 0.2(0.1,0.4)^{\mathrm{b}}$ & 58 & $6_{-5.2}^{+44} \times 10^{16 \mathrm{c}}$ & $2500_{-10}^{+10}[180](2520,2480)$ \\
\hline $\mathrm{C}_{3} \mathrm{H}_{4}$ & Compact $\mathrm{H}_{2} \mathrm{O}$ & $\sim 0.2(0.1,0.4)^{\mathrm{b}}$ & 92 & $1_{-0.8}^{+4} \times 10^{19 c}$ & $4400_{-5}^{+5}[240](4600,4330)$ \\
\hline $\mathrm{C}_{3} \mathrm{H}_{6}$ & Compact $\mathrm{H}_{2} \mathrm{O}$ & $\sim 0.2(0.1,0.4)^{\mathrm{b}}$ & 80 & $6_{-5.1}^{+34} \times 10^{18 c}$ & $3800_{-25}^{+20}[200](3810,3740)$ \\
\hline $\mathrm{C}_{3} \mathrm{H}_{8}$ & Compact $\mathrm{H}_{2} \mathrm{O}$ & $\sim 0.2(0.1,0.4)^{\mathrm{b}}$ & 73 & $4_{-3.4}^{+26} \times 10^{18 c}$ & $3500_{-5}^{+5}[200](3520,3490)$ \\
\hline $\mathrm{C}_{3} \mathrm{H}_{4}$ & Porous $\mathrm{H}_{2} \mathrm{O}$ & $\sim 1^{\mathrm{d}}$ & 97 & $1_{-0.8}^{+4} \times 10^{19 \mathrm{c}}$ & $4700_{-80}^{+70}[230]$ \\
\hline $\mathrm{C}_{3} \mathrm{H}_{6}$ & Porous $\mathrm{H}_{2} \mathrm{O}$ & $\sim 1^{\mathrm{d}}$ & 86 & $6_{-5.1}^{+34} \times 10^{18 c}$ & $4100_{-60}^{+50}[270]$ \\
\hline $\mathrm{C}_{3} \mathrm{H}_{8}$ & Porous $\mathrm{H}_{2} \mathrm{O}$ & $\sim 1^{\mathrm{d}}$ & 84 & $4_{-3.4}^{+26} \times 10^{18 c}$ & $4000_{-80}^{+60}[280]$ \\
\hline
\end{tabular}

Notes.

${ }^{\text {a }}$ Derived from IR band strengths, error is taken as $20 \%$.

${ }^{\mathrm{b}}$ Derived from integrated ion currents, error is taken as $50 \%$. Additional binding energies for different submonolayer coverages $(\sim 0.1,0.4)$ are provided in the $E_{b}$ column in parentheses.

${ }^{c}$ Pre-exponential factors for ices on $\mathrm{H}_{2} \mathrm{O}$ are derived from fitting the corresponding pure ice TPDs.

${ }^{\mathrm{d}}$ Assumed from TPD shape, error is taken as $50 \%$.

alkanes and alkynes off compact $\mathrm{H}_{2} \mathrm{O}$ by only $<10 \%$ (see below in Section 3.2). Because we are interested in the hydrocarbon $-\mathrm{H}_{2} \mathrm{O}$ interaction, we ran multiple compact TPDs of increasingly thin ices for each molecule until no interaction was visible, then took a slightly higher coverage as the chosen run to ensure that we had isolated the hydrocarbon- $\mathrm{H}_{2} \mathrm{O}$ interaction. We verified that the chosen runs had thicknesses of $\sim 0.2 \mathrm{ML}$ with the methods previously described in Section 2.2.

As with the pure and porous ices, the 2-carbon hydrocarbons have lower desorption temperatures when compared to the 3-carbon hydrocarbons. As for the porous ice experiments, both the porous 2- and 3-carbon hydrocarbon sets follow the desorption trend of alkane, alkene, alkyne.

\subsection{Binding Energies}

TPD curves and the resulting binding energy distributions for the 2- and 3-carbon hydrocarbons on $\mathrm{H}_{2} \mathrm{O}$ substrates are shown in Figures 2, 5, and 6. Derived binding energies and preexponential factors are listed in Table 2 . We obtain a range in binding energies of $2200-2800 \mathrm{~K}$ for pure 2-carbon hydrocarbon ices and a range of $3500-4200 \mathrm{~K}$ for pure 3-carbon hydrocarbon ices. Pre-exponential factors that resulted from fitting the pure ice TPD curves range from $\sim 4 \times$ $10^{15}-1 \times 10^{19} \mathrm{~s}^{-1}$ and were used to fit the corresponding TPD curves of hydrocarbons on porous and compact $\mathrm{H}_{2} \mathrm{O}$. While these pre-exponential factors are large, they are not unreasonable given that the hydrocarbons in our set are large; higher pre-exponential factors are correlated with larger molecular sizes because larger chain lengths result in higher

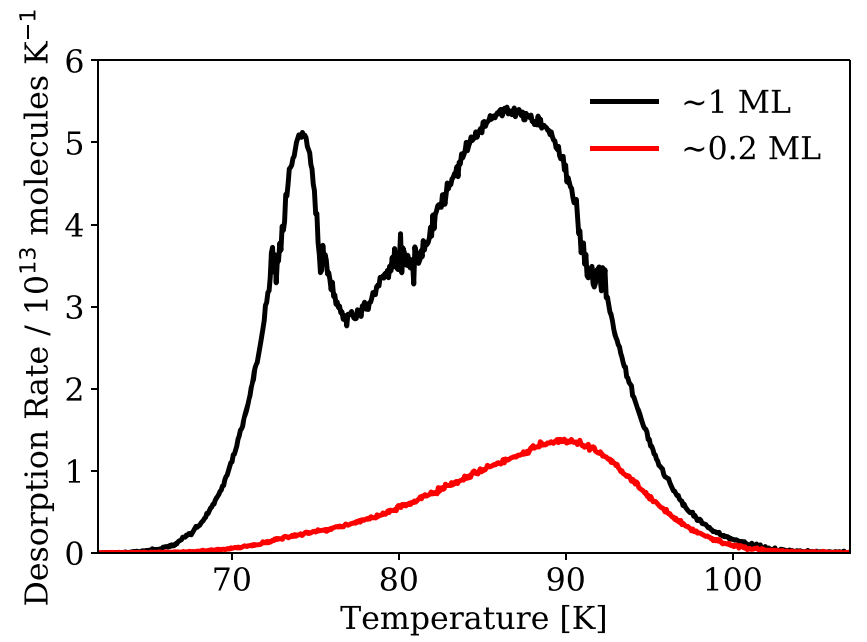

Figure 3. TPD curves of two different $\mathrm{C}_{3} \mathrm{H}_{6}$ coverages on porous $\mathrm{H}_{2} \mathrm{O}$ substrates. The loss of the multilayer peak from the first to the second TPD marks the transition from the multilayer to the submonolayer regime.

rotational entropy, which contributes to the pre-exponential factor calculation (Tait et al. 2005). In addition, Smith et al. (2016) report some multilayer $\mathrm{C}_{2} \mathrm{H}_{6}$ and $\mathrm{C}_{3} \mathrm{H}_{8}$ pre-exponential factors in the range of $10^{16}-10^{18} \mathrm{~s}^{-1}$, which is consistent with our results; though, they also report other pre-exponential factors up to $\sim 3 \times$ lower for thinner coverages. Ultimately, our derived pre-exponential factors may be too large, but we must adhere to them because they are derived in conjunction with the binding energies, and we emphasize that they are the proper 


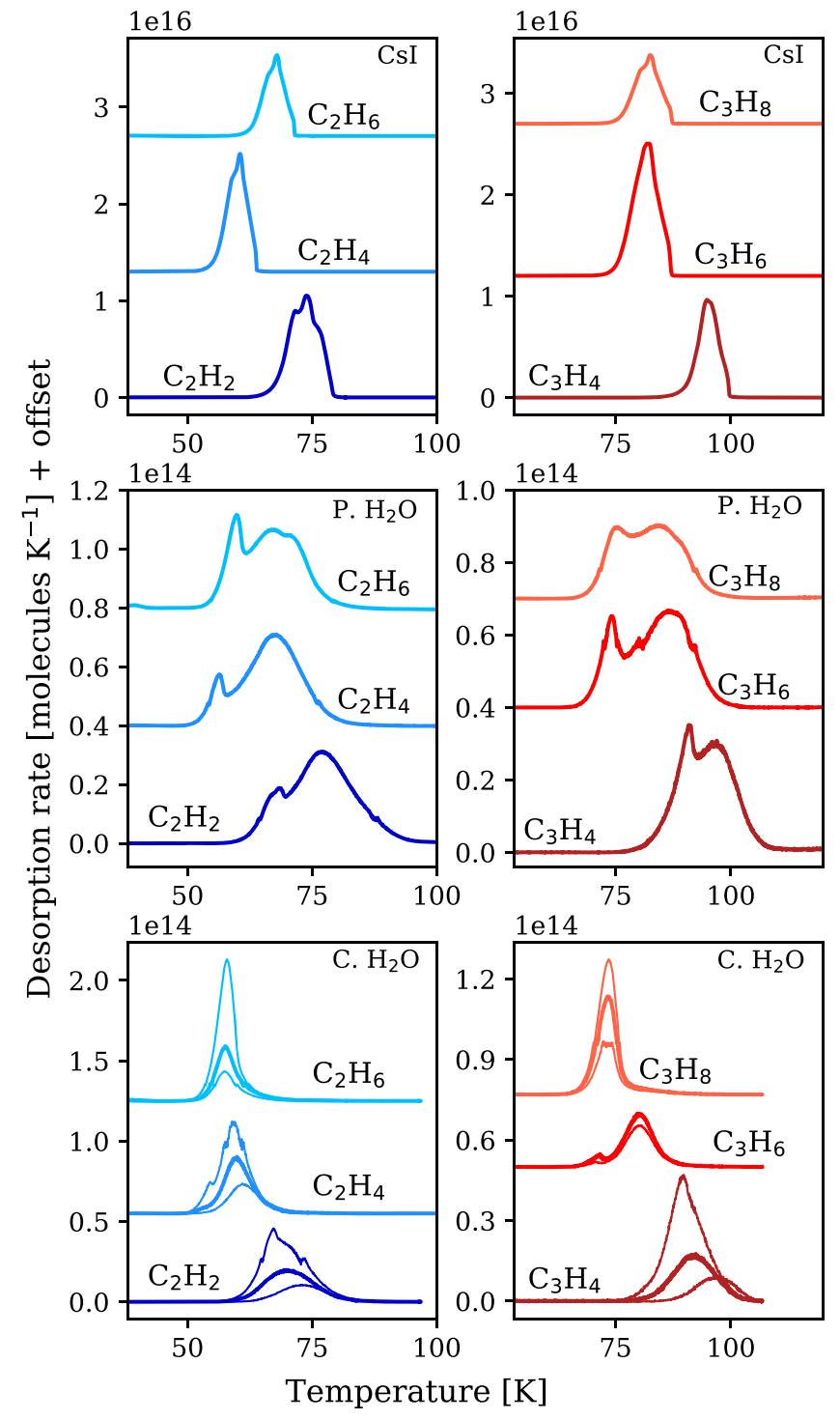

Figure 4. All TPD curves, with TPDs of Cs I substrates displayed in the top panels, TPDs of porous $\mathrm{H}_{2} \mathrm{O}$ substrates displayed in the middle panels, and TPDs of compact $\mathrm{H}_{2} \mathrm{O}$ substrates displayed in the bottom panels. The TPDs on compact $\mathrm{H}_{2} \mathrm{O}$ are overplotted with runs of slightly higher and lower ice coverages for comparison (thinner lines). For all hydrocarbons other than $\mathrm{C}_{2} \mathrm{H}_{6}$ and $\mathrm{C}_{3} \mathrm{H}_{8}$, the higher coverage runs feature multilayer peaks that disappear as coverage decreases. The significantly larger desorption rate scale for the pure TPDs compared to the porous and compact TPDs is due to correspondingly larger coverages/amounts of molecules being desorbed. For zeroth-order desorption, the desorption peak shifts to higher temperatures with increasing coverage, which is why the multilayer peaks for the pure and porous TPDs do not perfectly align.

pre-exponential factors that should be used in chemical models. The uncertainties on the pre-exponential factors are from the $2 \mathrm{~K}$ absolute error on the temperature instrument (Table 2).

The alkyne and alkene binding energies from desorption off compact $\mathrm{H}_{2} \mathrm{O}$ substrates are $\sim 5 \%-10 \%$ higher than the pure hydrocarbon binding energies. The compact alkane binding energies are consistent with the pure binding energies. Lower binding energies for compact ices than pure ices have been observed in the literature for some species, such as atomic oxygen and $\mathrm{O}_{2}$ (Noble et al. 2012; He et al. 2015). To check that picking coverages of specifically $\sim 0.2 \mathrm{ML}$ on compact ices did not result in biased binding energy distributions, we derived distributions for additional experiments of $\sim 0.1-0.4$ ice coverages on compact ices, and found good agreement between centroids for all coverages (binding energy variation is $\sim 200 \mathrm{~K}$ at most; Table 2). For the binding energy distributions, see Figure 11 in the Appendix. Thus, we conclude that varying ice thicknesses does not affect binding energies significantly if the difference is below a monolayer.

Binding energy values increase when moving from compact to porous $\mathrm{H}_{2} \mathrm{O}$ substrate experiments; the binding energies for hydrocarbons desorbing off porous $\mathrm{H}_{2} \mathrm{O}$ are $\sim 5 \%-20 \%$ higher $(300-500 \mathrm{~K})$ than those of hydrocarbons desorbing off compact $\mathrm{H}_{2} \mathrm{O}$. This is expected as diffusion of adsorbate species into substrate pores leads to availability of higher energy binding sites (e.g., Hornekær et al. 2005; Zubkov et al. 2007; Karssemeijer et al. 2014). This shift should be considered a lower limit because binding energies for ices on $\mathrm{H}_{2} \mathrm{O}$ substrates decrease with coverage (Noble et al. 2012), and the porous experiments have substantially higher ice coverages than the compact experiments.

The only binding energies that exist for this set of hydrocarbons in the literature are reported in Smith et al. (2016) for $\mathrm{C}_{2} \mathrm{H}_{6}$ and $\mathrm{C}_{3} \mathrm{H}_{8}$ on compact $\mathrm{H}_{2} \mathrm{O}$. Smith et al. (2016) report binding energies of $2500 \mathrm{~K}$ and $3200 \mathrm{~K}$ for $\mathrm{C}_{2} \mathrm{H}_{6}$ and $\mathrm{C}_{3} \mathrm{H}_{8}$ on compact $\mathrm{H}_{2} \mathrm{O}$ ice, respectively, which agree well with the binding energies we obtain from our experiments (Table 2). However, more data exist on sublimation enthalpies that can be used to calculate binding energies. A compendium of sublimation enthalpies is reported in Acree \& Chickos (2016) for a large set of compounds that includes all hydrocarbons used in this study except $\mathrm{C}_{3} \mathrm{H}_{4}$. When these are converted to binding energies, they also agree well with those obtained from our experiments.

The uncertainties on binding energy values are from the errors in ice thickness and the $2 \mathrm{~K}$ absolute error on the temperature instrument. The $2 \mathrm{~K}$ error is only relevant in the case of pure binding energy values as the FWHM values of the binding energy distributions for the monolayer and submonolayer experiments are always greater than the uncertainties from the $2 \mathrm{~K}$ error (Table 2). We verified that the ice thickness errors have little effect on the binding energies for the pure ices by taking thickness errors of up to $50 \%$ and noting only negligible shifts in resultant binding energies and derived pre-exponential values.

\section{Discussion}

We present the binding energies for $\mathrm{C}_{2} \mathrm{H}_{2}, \mathrm{C}_{2} \mathrm{H}_{4}, \mathrm{C}_{2} \mathrm{H}_{6}$, $\mathrm{C}_{3} \mathrm{H}_{4}, \mathrm{C}_{3} \mathrm{H}_{6}$, and $\mathrm{C}_{3} \mathrm{H}_{8}$ from both pure and off porous and compact $\mathrm{H}_{2} \mathrm{O}$ substrates. We observe a clear increase in the binding energies between the 2- and 3-carbon hydrocarbons, and the binding energies for the 2-carbon hydrocarbons are higher than the $\mathrm{CH}_{4}$ binding energies in similar ice environments, presenting a clear trend (He et al. 2016; Smith et al. 2016).

Within the 2- and 3-carbon families and across all ices, the alkanes and alkenes have similar binding energies while the alkyne binding energies are noticeably higher. This is at odds with the assumption that desorption temperatures/binding energies scale with molecular weight, which is sometimes used in astrochemical simulations when experimental data is lacking (Garrod et al. 2008).

For the set of hydrocarbons analyzed, the compact $\mathrm{H}_{2} \mathrm{O}$-hydrocarbon interactions are only slightly stronger $(\sim 5 \%-10 \%)$ than the hydrocarbon-hydrocarbon interactions. 

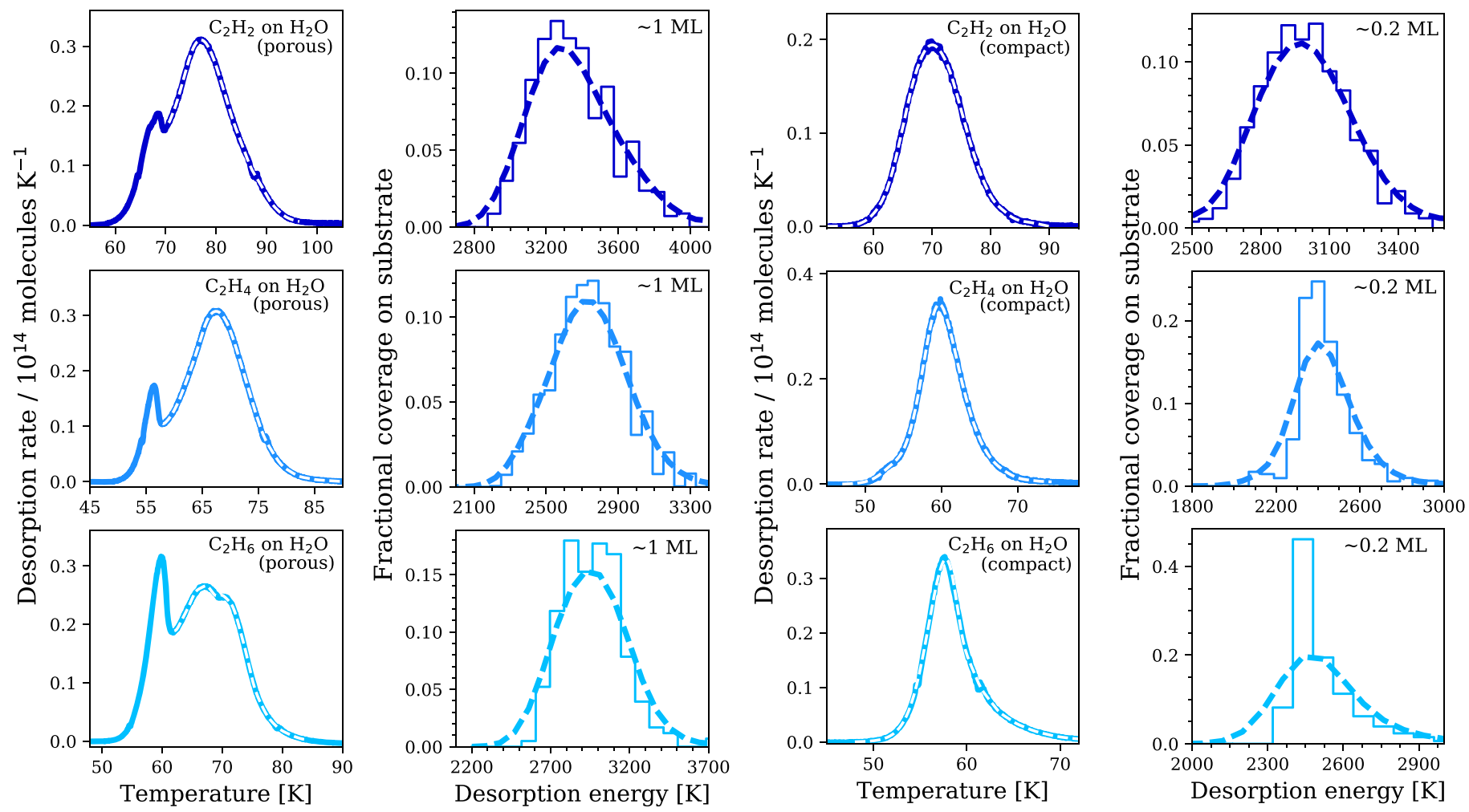

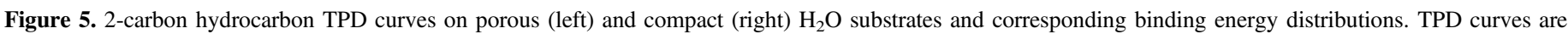

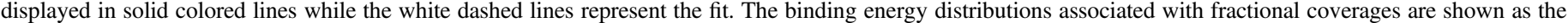
histograms, while the dashed lines represent the smoothed distributions using a Gaussian filter for clarity.

These results differ from those of studies that constrained the effects of compact $\mathrm{H}_{2} \mathrm{O}$ on the binding energies of other molecules, such as $\mathrm{CO}$ and $\mathrm{N}_{2}$; Fayolle et al. (2016) determined that the binding energies of $\mathrm{CO}$ and $\mathrm{N}_{2}$ interactions with compact $\mathrm{H}_{2} \mathrm{O}$ substrates were $30 \%-50 \%$ larger than the binding energies of $\mathrm{CO}$ and $\mathrm{N}_{2}$ in pure ices when considering similar ice coverages.

The largest binding energies are achieved on porous $\mathrm{H}_{2} \mathrm{O}$ substrates, which is consistent with previous studies of $\mathrm{CO}$ and $\mathrm{N}_{2}$ ices on $\mathrm{H}_{2} \mathrm{O}$ (Fayolle et al. 2016; He et al. 2016). However, the increase in binding energies $(\sim 10 \%-20 \%)$ from hydrocarbon-hydrocarbon interactions to porous $\mathrm{H}_{2} \mathrm{O}$-hydrocarbon interactions is again relatively low compared to the $\sim 80 \%$ increase of $\mathrm{CO}$ and $\mathrm{N}_{2}$ on porous $\mathrm{H}_{2} \mathrm{O}$ (Fayolle et al. 2016). The relatively low shifts in binding energies for the hydrocarbons on $\mathrm{H}_{2} \mathrm{O}$ can be explained by their hydrophobic nature, which allows attachment to the $\mathrm{H}_{2} \mathrm{O}$ substrate via only weak interactions.

Binding variations for alkanes, alkenes, and alkynes with $\mathrm{H}_{2} \mathrm{O}$ may arise from differences in hydrocarbon molecular geometry (size, linearity) due to different bonding structures (single, double, or triple bonds) that create different charge densities and orbital hybridizations. Such steric and electronic effects may impact how the hydrocarbons interact with $\mathrm{H}_{2} \mathrm{O}$. The 2- and 3-carbon family desorption temperature trend of the alkynes exhibiting higher desorption temperatures than the alkenes and alkynes (see Figure 7) is not obvious, and will require theoretical studies to elucidate.

\section{Astrophysical Implications}

We use our newly derived binding energies to estimate the sublimation front locations of all six hydrocarbons in a representative protoplanetary disk, characterized by a disk temperature profile $T=200 \mathrm{~K} \times(r / 1 \mathrm{au})^{-0.62}$. This is the median disk temperature profile derived from a sample of 24 circumstellar disks in the Taurus-Auriga and OphiuchusScorpius star-forming regions (Andrews \& Williams 2007).

Because sublimation front locations are set by sublimation temperatures, we use the prescription from Hollenbach et al. (2009) to calculate sublimation temperatures from our binding energies, which is derived by equating the flux of adsorbing and desorbing molecules off a grain surface:

$$
T_{i} \simeq\left(E_{i} / k\right)\left[\ln \left(\frac{4 N_{i} f_{i} \nu_{i}}{n_{i} v_{i}}\right)\right]^{-1},
$$

where $T_{i}$ is the sublimation temperature, $E_{i}$ is the binding energy of species $i, N_{i}$ is the number of adsorption sites per $\mathrm{cm}^{2}$ $\left(N_{i} \sim 10^{15}\right.$ cites $\left.\mathrm{cm}^{2}\right), f_{i}$ is the fraction of the surface adsorption sites that are occupied by species $i, n_{i}$ is the gas-phase number density of species $i, v_{i}$ is its thermal speed, and $\nu_{i}$ is the vibrational frequency for which we use our derived preexponential factors.

To estimate $f_{i}$, we rely on cometary abundances with respect to $\mathrm{H}_{2} \mathrm{O}$ because there are no hydrocarbon abundances for protoplanetary disks available. The observed abundances of $\mathrm{C}_{2} \mathrm{H}_{2}$ and $\mathrm{C}_{2} \mathrm{H}_{6}$ in cometary ices are $0.2 \%-0.6 \%$ with respect to $\mathrm{H}_{2} \mathrm{O}$ (Mumma \& Charnley 2011). We further adopt abundances of $0.1 \%$ for $\mathrm{C}_{2} \mathrm{H}_{4}$, and $0.01 \%$ for the 3-carbon hydrocarbons with respect to $\mathrm{H}_{2} \mathrm{O}$, assuming that cometary abundances decrease by an order of magnitude from $\mathrm{CH}_{4}(1 \%$ with respect to $\mathrm{H}_{2} \mathrm{O}$ ) to $\mathrm{C}_{2} \mathrm{H}_{X}$, and from $\mathrm{C}_{2} \mathrm{H}_{X}$ to $\mathrm{C}_{3} \mathrm{H}_{X}$. As cometary ice is $\sim 80 \% \mathrm{H}_{2} \mathrm{O}$ in composition (Delsemme 1988), we calculate $f_{i}$ for each hydrocarbon by multiplying the 

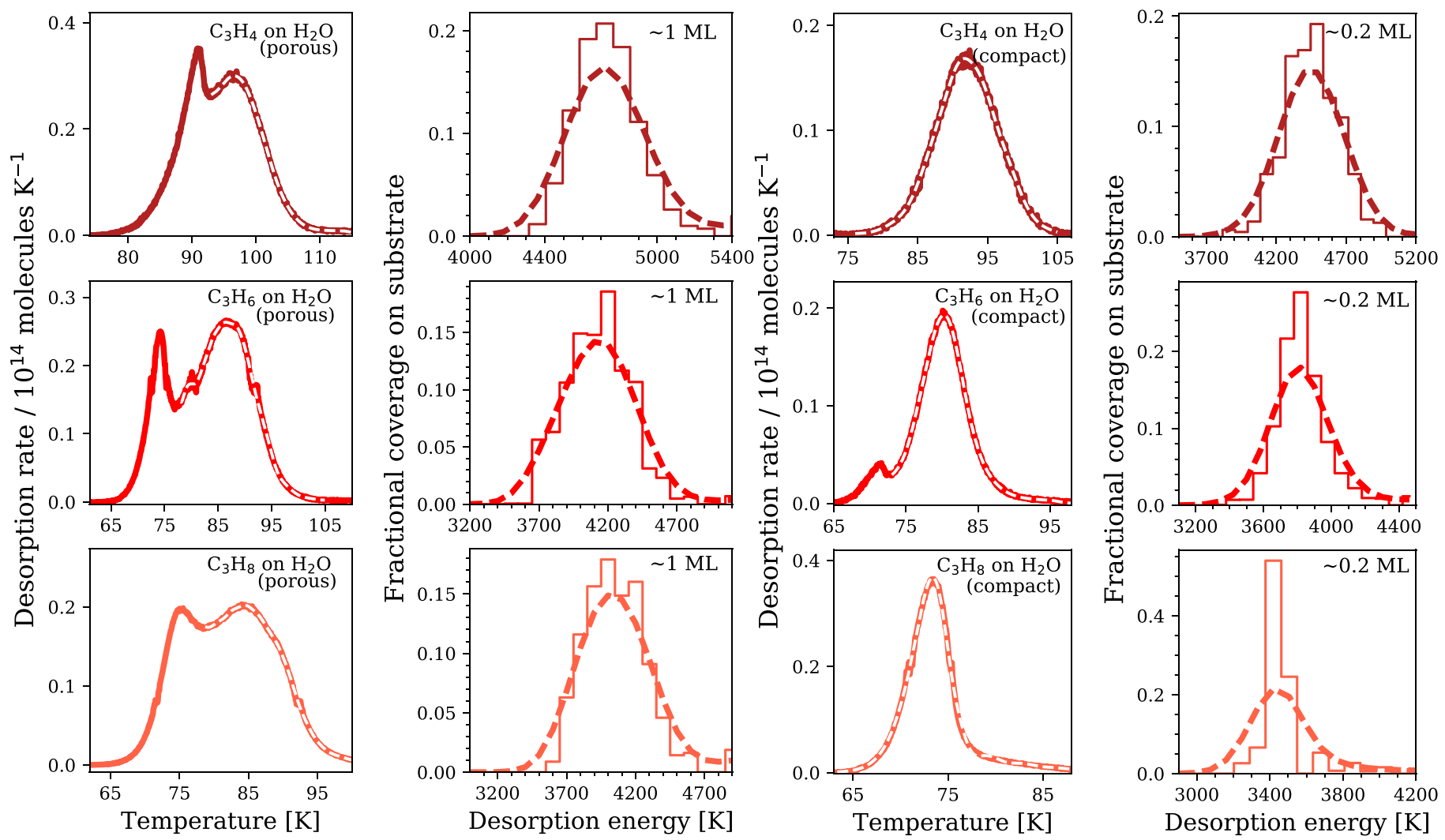

Figure 6. 3-carbon hydrocarbon TPD curves on porous (left) and compact (right) $\mathrm{H}_{2} \mathrm{O}$ substrates and corresponding binding energy distributions, similar to Figure 5 .

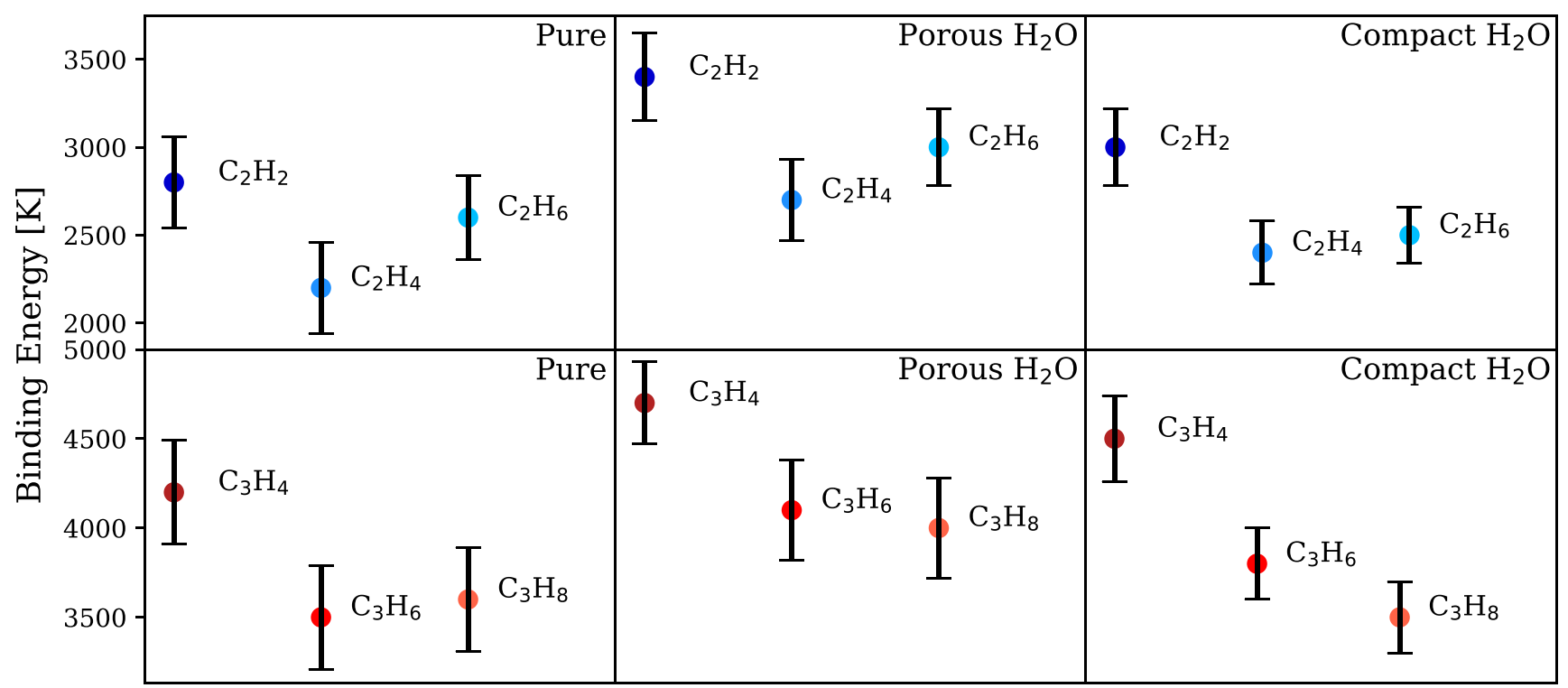

Figure 7. Binding energy values of all TPD experiments. Pure ice binding energy are displayed in the left panels, porous $\mathrm{H}_{2} \mathrm{O}$ substrate binding energies are displayed in the middle panels, and compact $\mathrm{H}_{2} \mathrm{O}$ substate binding energies are displayed in the right panels.

cometary hydrocarbon abundance with respect to water by 0.8 to obtain an estimate of the hydrocarbon surface coverage fraction in icy grain mantles.

We estimate the gas-phase number density $n_{i}$ by multiplying the number density of atomic hydrogen in protoplanetary disks (which can be regarded as overall density), the $\mathrm{H}_{2} \mathrm{O}$ abundance with respect to hydrogen, and the cometary hydrocarbon abundance with respect to $\mathrm{H}_{2} \mathrm{O}$. We take the number density of hydrogen in disks to be $10^{10} \mathrm{~cm}^{-3}$ in the midplane (e.g., Öberg et al. 2011), and the $\mathrm{H}_{2} \mathrm{O}$ abundance to be $10^{-4}$ per $\mathrm{H}$-atom (e.g., Boogert et al. 2015).

We then use the disk temperature profile to estimate sublimation front locations from the sublimation temperatures. We find that the 2-carbon hydrocarbons desorb between 6 and $11 \mathrm{au}$, or $\sim 70 \mathrm{~K}$ and $\sim 50 \mathrm{~K}$, while the 3-carbon hydrocarbons desorb between 4 and $6 \mathrm{au}$, or $\sim 80 \mathrm{~K}$ and $\sim 70 \mathrm{~K}$. If we limit 


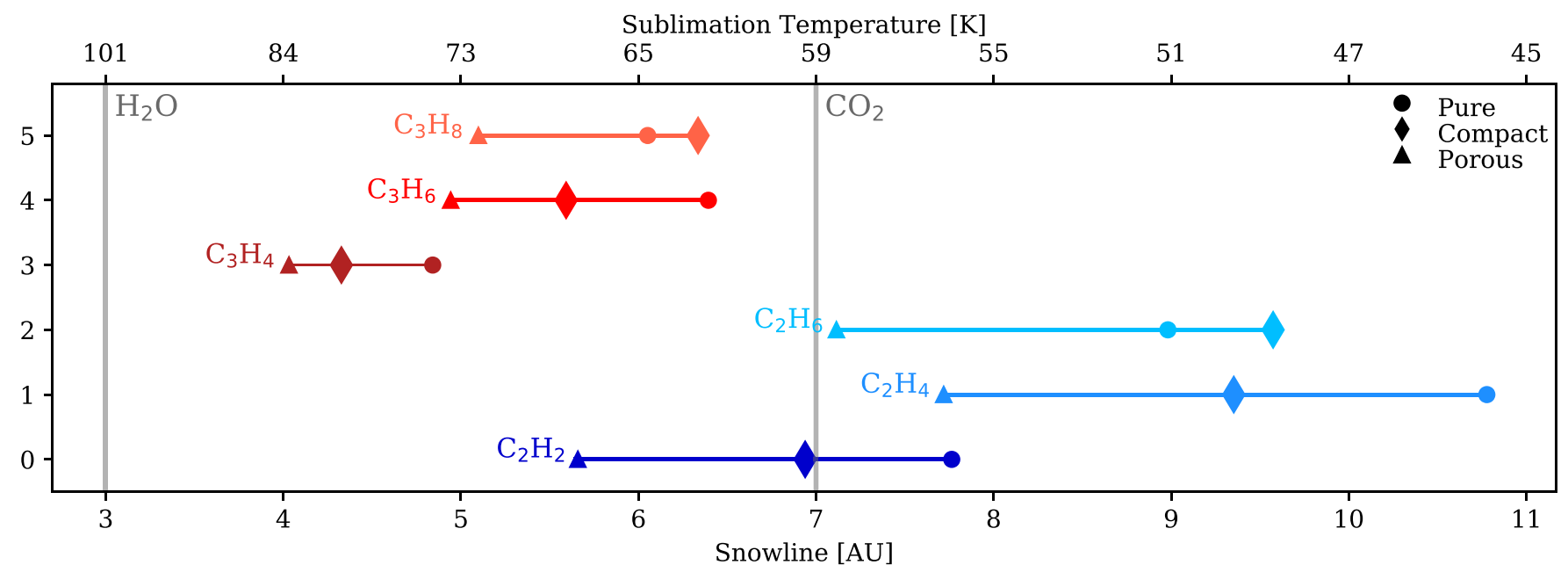

Figure 8. Sublimation front locations calculated from binding energies for each hydrocarbon. Binding energies (porous, compact, and pure) are provided above each sublimation front illustration. Sublimation fronts for $\mathrm{H}_{2} \mathrm{O}$ (pure ice) and $\mathrm{CO}_{2}$ (in nonporous amorphous $\mathrm{H}_{2} \mathrm{O}$-dominated ice) are provided for reference. The $\mathrm{H}_{2} \mathrm{O}$ and $\mathrm{CO}_{2}$ binding energies used for the sublimation front calculations are taken from Fraser et al. (2001) and Noble et al. (2012), respectively.

ourselves to the most likely case of desorption from grains with compact $\mathrm{H}_{2} \mathrm{O}$ mantles, these ranges shrink to 6 and 8 au for 2-carbon hydrocarbons, and 4 and 6 au for 3-carbon hydrocarbons (see Figure 8).

Assuming negligible hydrocarbon entrapment in $\mathrm{H}_{2} \mathrm{O}$ ice, any solid bodies that form within 4 au will be depleted in small hydrocarbons. This potentially limits their ability to participate in ice chemistry and the organic chemistry of planets that they contribute to. In contrast, planetesimals and planets that form outside 8 au will be rich in hydrocarbons. This includes comets, which can migrate and deliver material to other bodies throughout the disk. However, entrapment is certainly possible, and future studies on entrapment efficiencies of different hydrocarbons are needed to obtain a complete picture of the distributions of small hydrocarbons during planet formation.

\section{Conclusions}

In this study, we obtained binding energies $\mathrm{C}_{2} \mathrm{H}_{2}, \mathrm{C}_{2} \mathrm{H}_{4}$, $\mathrm{C}_{2} \mathrm{H}_{6}, \mathrm{C}_{3} \mathrm{H}_{4}, \mathrm{C}_{3} \mathrm{H}_{6}$, and $\mathrm{C}_{3} \mathrm{H}_{8}$ in both pure ices and off porous and compact $\mathrm{H}_{2} \mathrm{O}$. We found:

1. The binding energies of pure 2- and 3-carbon amorphous ices range from 2200 to $2800 \mathrm{~K}$ and 3500 to $4200 \mathrm{~K}$, respectively.

2 . In the submonolayer regime, the binding energies of 2and 3-carbon amorphous ices off compact $\mathrm{H}_{2} \mathrm{O}$ substrates range from 2400 to $3000 \mathrm{~K}$ and 3500 to $4400 \mathrm{~K}$, respectively. Off porous $\mathrm{H}_{2} \mathrm{O}$ substrates, the binding energies of 2- and 3-carbon amorphous ices range from 2800 to $3400 \mathrm{~K}$ and 4000 to $4700 \mathrm{~K}$, respectively. These porous binding energies are $\sim 10 \%-20 \%$ larger than the pure ice binding energies.
3. Within the 2- and 3-carbon hydrocarbon sets, the alkynes (i.e., least-saturated) hydrocarbons exhibit the largest binding energies.

From these results, we can draw the following conclusions:

1. There is a relatively small difference in binding energies between pure hydrocarbon ices and hydrocarbon ices desorbing off $\mathrm{H}_{2} \mathrm{O}$ compared to what has been measured for other volatile species $\left(\mathrm{CO}, \mathrm{N}_{2}\right)$. This implies that $\mathrm{H}_{2} \mathrm{O}$ has a small influence on the snowline locations of these hydrocarbons in protoplanetary disks.

2. Though the alkynes $\left(\mathrm{C}_{2} \mathrm{H}_{2}\right.$ and $\left.\mathrm{C}_{3} \mathrm{H}_{4}\right)$ are the smallest molecules within the 2- and 3-carbon hydrocarbon sets, they exhibit higher binding energies, demonstrating that molecular size does not necessarily correlate with larger desorption temperatures/binding energies within molecular families.

A.B. acknowledges funding from the Origins of Life Initiative at Harvard. E.C.F.'s contribution was partly carried out at the Jet Propulsion Laboratory, California Institute of Technology, under a contract with the National Aeronautics and Space Administration. K.I.Ö. acknowledges funding from the Simons Collaboration on the Origins of Life Investigator award \#321183.

\section{Appendix Supplementary Figures}

The following supplementary figures are included: Figures 9, 10 , and 11. 

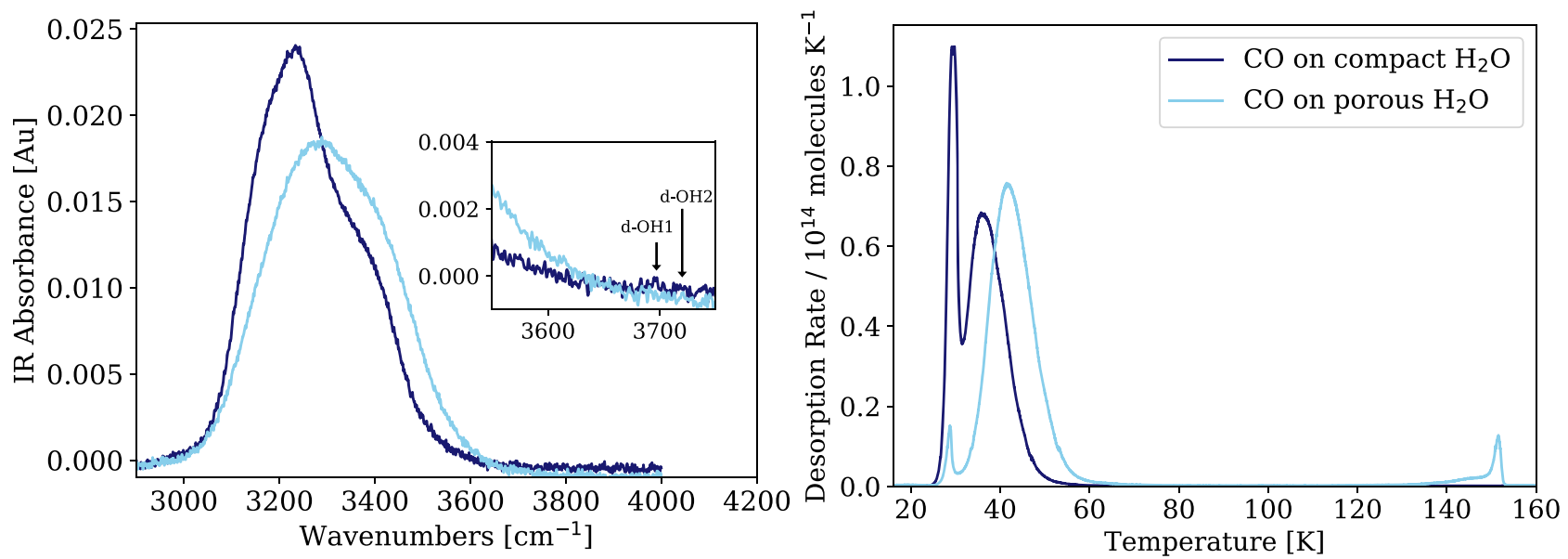

Figure 9. Left: FTIR data for one representative porous and one compact $\mathrm{H}_{2} \mathrm{O}$ ice substrate, with an inset centered on where the dangling O-H bond features would be located (d-O-H1 at $\sim 3697 \mathrm{~cm}^{-1}$, d-O-H2 at $\sim 3720 \mathrm{~cm}^{-1}$ ). The degree of porosity cannot be determined from the dangling O-H bond feature as it is not visible in the spectra. Right: TPD curves of $\mathrm{CO}$ on compact and porous $\mathrm{H}_{2} \mathrm{O}$ substrates. There is a CO desorption peak around the $\mathrm{H}_{2} \mathrm{O}$ desorption temperature for the porous substrate experiment, but not for the compact substrate experiment.

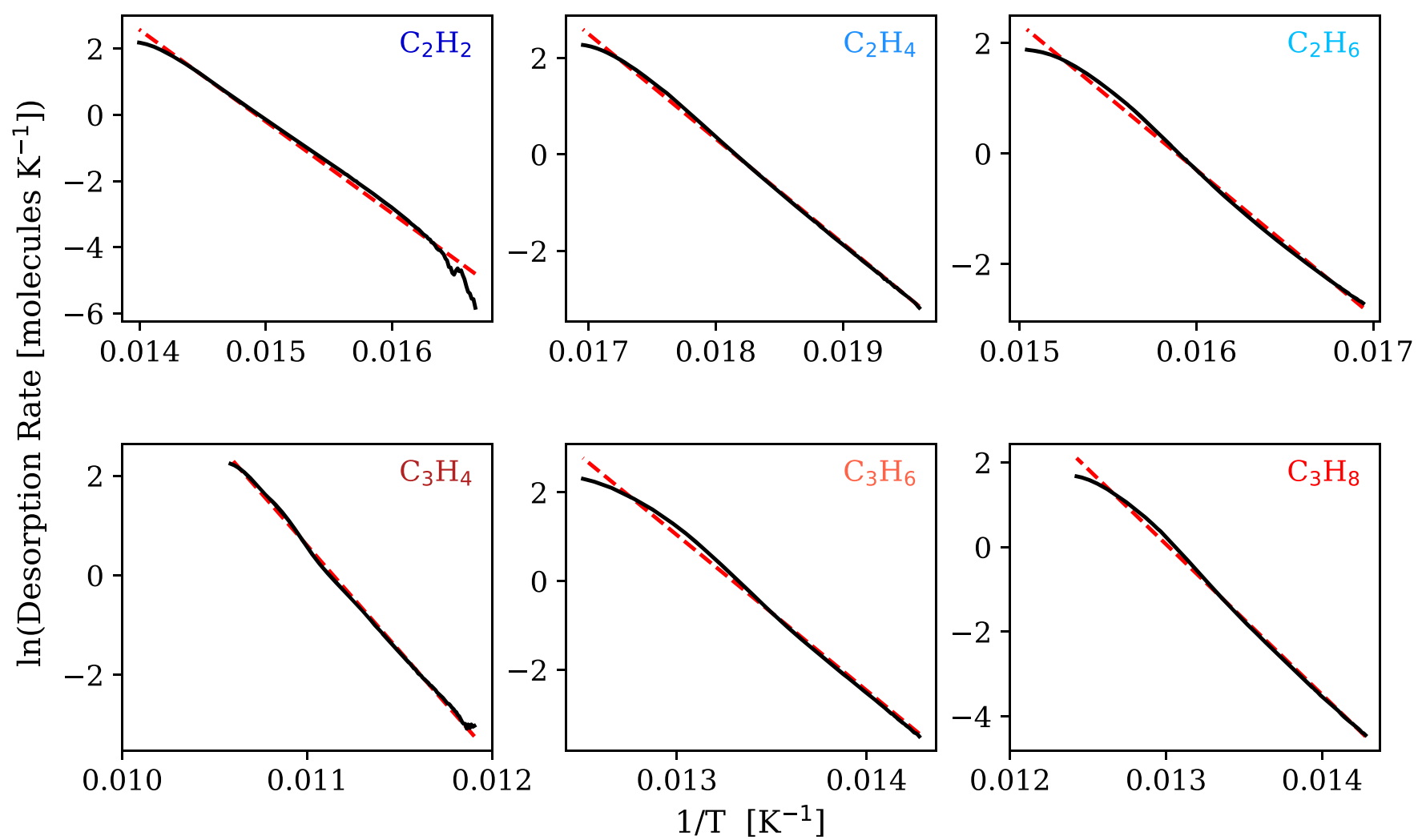

Figure 10. Arrhenius plots for all pure TPDs with fits to determine the binding energies $E_{b}$ and pre-exponential factors $\nu$. The desorption rate data are represented by the black lines, while the fits are represented by the dashed red lines. 

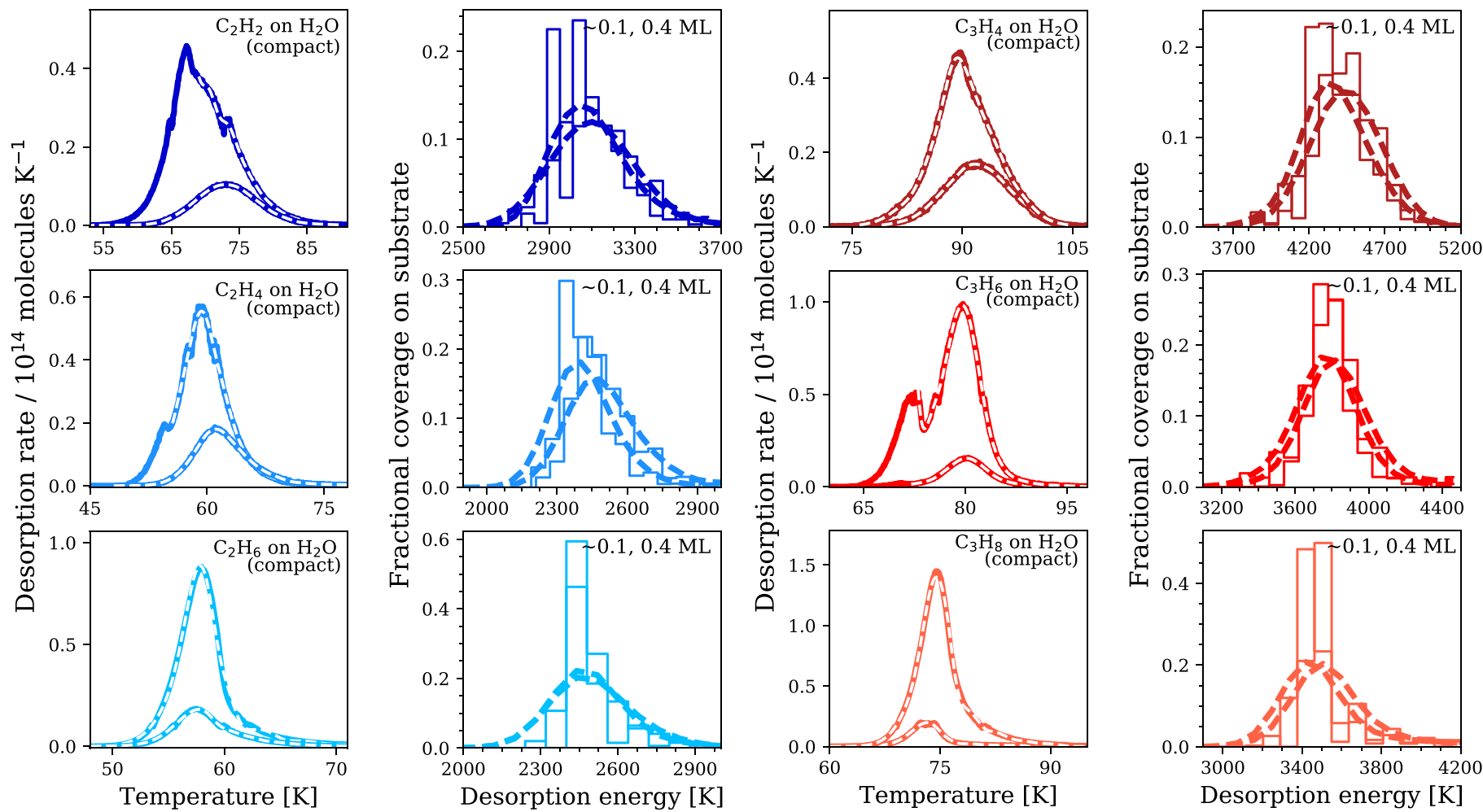

Figure 11. 2- and 3-carbon hydrocarbon TPD curves on compact $\mathrm{H}_{2} \mathrm{O}$ substrates (coverages of $\sim 0.1,0.4 \mathrm{ML}$ ) and corresponding binding energy distributions, similar to Figures 5 and 6 . The variation in binding energy distribution centroids for coverages between $\sim 0.1,0.2$ (target coverage), and 0.4 ML is less than $200 \mathrm{~K}$ for all hydrocarbons used in this study.

\section{ORCID iDs}

Aida Behmard (ib https://orcid.org/0000-0003-0012-9093

Edith C. Fayolle iㅣ https://orcid.org/0000-0001-8109-5256

Dawn M. Graninger (1) https://orcid.org/0000-0003-

1582-0581

Jennifer B. Bergner (ํ) https://orcid.org/0000-0002-8716-0482

Karin I. Öberg (1) https://orcid.org/0000-0001-8798-1347

\section{References}

Acree, W., \& Chickos, J. S. 2016, JPCRD, 45, 3

Agúndez, M., \& Wakelam, V. 2013, ChRv, 113, 8710

Anderson, A., Andrews, B., \& Torrie, B. H. 1985, JRSp, 16, 202

Andrews, S. M., \& Williams, J. P. 2007, ApJ, 659, 705

Bernstein, M. P., Sandford, S. A., \& Allamandola, L. J. 2005, ApJS, 161, 53

Bertin, M., Romanzin, C., Michaut, X., Jeseck, P., \& Fillion, J. H. 2011, J. Phys. Chem. C, 115, 12920

Betz, A. L. 1981, ApJL, 244, L103

Boogert, A. C. A., Gerakines, P. A., \& Whittet, D. C. B. 2015, ARA\&A, 53,541

Bossa, J.-B., Isokoski, K., de Valois, M. S., \& Linnartz, H. 2012, A\&A, 545, A82

Bossa, J.-B., Isokoski, K., Paardekooper, D. M., et al. 2014, A\&A, 561, A136 Brooke, T. Y., Tokunaga, A. T., Weaver, H. A., et al. 1996, Natur, 383, 606 Charnley, S. B. 2004, AdSpR, 33, 23

Cleeves, L. I., Bergin, E. A., Alexander, C. M. O. D. F., et al. 2016, ApJ, 819,13

Clements, A. R., Berk, B., Cooke, I. R., \& Garrod, R. T. 2018, PCCP, 20, 5553 Collings, M. P., Anderson, M. A., Chen, R., et al. 2004, MNRAS, 354, 1133

Collings, M. P., Frankland, V. L., Lasne, J., et al. 2015, MNRAS, 449, 1826 Delsemme, A. H. 1988, RSPTA, 325, 509

Devlin, J. P., \& Buch, V. 1995, JPhCh, 99, 16534

Doronin, M., Bertin, M., Michaut, X., Phillipe, L., \& Fillion, J. H. 2015, $\mathrm{JChPh}, 143,084703$

Fayolle, E. C., Balfe, J., Loomis, R., et al. 2016, ApJL, 816, L28
Fraser, H. J., Collings, M. P., McCoustra, M. R. S., \& Williams, D. A. 2001, MNRAS, 327, 1165

Garrod, R. T., Widicus Weaver, S. L., \& Herbst, E. 2008, ApJ, 682, 283

Gerakines, P. A., Schutte, W. A., Greenberg, J. M., \& van Dishoeck, E. F. 1995, A\&A, 296, 810

Gibb, E. L., \& Horne, D. 2013, ApJL, 776, L28

Gibb, E. L., Van Brunt, K. A., Brittain, S. D., \& Rettig, T. W. 2007, ApJ, 660, 1572

Graninger, D. M., Wilkins, O. H., \& Öberg, K. I. 2016, ApJ, 819, 140

Guzmán, V. V., Pety, J., Goicoechea, J. R., et al. 2015, ApJL, 800, L33

Hagen, W., Tielens, A. G. G. M., \& Greenberg, J. M. 1981, CP, 56, 367

Hardegree-Ullman, E. E., Gudipati, M. S., Boogert, A. C. A., et al. 2014, ApJ, 784,172

He, J., Acharyya, K., \& Vidali, G. 2016, ApJ, 825, 89

He, J., Emtiaz, S. M., \& Vidali, G. 2017, ApJ, 851, 104

He, J., Shi, J., Hopkins, T., Vidali, G., \& Kaufman, M. J. 2015, ApJ, 801, 120

Hollenbach, D., Kaufman, M. J., Bergin, E. A., \& Melnick, G. J. 2009, ApJ, 690,1497

Hornekær, L., Baurichter, A., Petrunin, V. V., et al. 2005, JChPh, 122, 124701

Hudson, R. L., Ferrante, R. F., \& Moore, M. H. 2014a, Icar, 228, 276

Hudson, R. L., Gerakines, P. A., \& Moore, M. H. 2014b, Icar, 243, 148

Hudson, R. L., \& Moore, M. H. 1997, Icar, 126, 233

Isokoski, K., Bossa, J.-B., Triemstra, T., \& Linnartz, H. 2014, PCCP, 16, 3456

Kaiser, R. I., \& Roessler, K. 1998, ApJ, 503, 959

Karssemeijer, L. J., Ioppolo, S., van Hemert, M. C., et al. 2014, ApJ, 781, 16

Kastner, J. H., Qi, C., Gorti, U., et al. 2015, ApJ, 806, 75

Kawakita, H., Dello Russo, N., Vervack, R., Jr., et al. 2014, ApJ, 788, 110

Khanna, R. K., Ospina, M. J., \& Zhao, G. 1988, Icar, 73, 527

Lahuis, F., van Dishoeck, E. F., Boogert, A. C. A., et al. 2006, ApJL, 636, L145

Lauck, T., Karssemeijer, L., Shulenberger, K., et al. 2015, ApJ, 801, 118

Markwick, A. J., Ilgner, M., Millar, T. J., \& Henning, T. 2002, A\&A, 385, 632

Mastrapa, R. M., Sandford, S. A., Roush, T. L., Cruikshank, D. P., \& Dalle Ore, C. M. 2009, ApJ, 701, 1347

Moore, M. H., \& Hudson, R. L. 1998, Icar, 135, 518

Mumma, M. J., \& Charnley, S. B. 2011, ARA\&A, 49, 471

Mumma, M. J., Disanti, M. A., dello Russo, N., et al. 1996, Sci, 272, 1310

Noble, J. A., Congiu, E., Dulieu, F., \& Fraser, H. J. 2012, MNRAS, 421, 768 
Oba, Y., Miyauchi, N., Hidaka, H., et al. 2009, ApJ, 701, 464

Öberg, K. I., Boogert, A. C. A., Pontoppidan, K. M., et al. 2008, ApJ, 678, 1032

Öberg, K. I., Murray-Clay, R., \& Bergin, E. A. 2011, ApJL, 743, L16

Öberg, K. I., van Dishoeck, E. F., Linnartz, H., \& Andersson, S. 2010, ApJ, 718,832

Pontoppidan, K. M., Salyk, C., Bergin, E. A., et al. 2014, in Protostars and Planets VI, ed. H. Beuther et al. (Tucson, AZ: Univ. Arizona Press), 363

Qi, C., Öberg, K. I., Wilner, D. J., \& Rosenfeld, K. A. 2013, ApJL, 765, L14

Raut, U., Famá, M., Teolis, B. D., \& Baragiola, R. A. 2007, JChPh, 127, 204713

Sakai, N., \& Yamamoto, S. 2013, ChRv, 113, 8981
Smith, R. S., May, R. A., \& Kay, B. D. 2016, JPCB, 120, 1979

Snyder, L. E., \& Buhl, D. 1973, NPhS, 243, 45

Tait, S. L., Dohnálek, Z., Campbell, C. T., \& Kay, B. D. 2005, JChPh, 122, 164708

Thi, W.-F., Ménard, F., Meeus, G., et al. 2011, A\&A, 530, L2

Tielens, A. G. G. M., \& Hagen, W. 1982, A\&A, 114, 245

Tucker, K. D., Kutner, M. L., \& Thaddeus, P. 1974, ApJL, 193, L115

Viti, S., Collings, M. P., Dever, J. W., McCoustra, M. R. S., \& Williams, D. A. 2004, MNRAS, 354, 1141

Zhao, G., Ospina, M. J., \& Khanna, R. K. 1988, AcSpA, 44, 27

Zubkov, T., Smith, R. S., Engstrom, T. R., \& Kay, B. D. 2007, JChPh, 127, 184707 